\title{
SERUM VASCULAR ENDOTHELIAL GROWTH FACTOR LEVELS IN NORMAL EARLY PREGNANCIES AND RECURRENT ABORTION PATIENTS
}

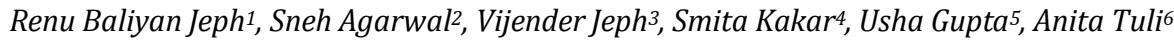

${ }_{1}^{1}$ Associate Professor, Department of Anatomy, Vardhaman Mahavir Medical College and SJH, New Delhi, India.

2 Director Professor and HOD, Department of Anatomy, Lady Hardinge Medical College, New Delhi, India.

${ }_{3}^{3}$ Associate Professor, Department of Pathology, Santosh Medical College, Ghaziabad, Uttar Pradesh, India.

${ }^{4}$ Ex-Director Professor, Department of Anatomy, Lady Hardinge Medical College, New Delhi, India.

${ }^{5}$ Ex-Director Professor, Department of Obstetrics and Gynaecology, Lady Hardinge Medical College, New Delhi, India.

${ }^{6}$ Director Professor, Department of Anatomy, Lady Hardinge Medical College, New Delhi, India.

\section{BACKGROUND}

ABSTRACT

Adequate angiogenesis is required for successful continuation of pregnancy and Vascular Angiogenic Growth Factor (VEGF) is one of the most important pro-angiogenic factors regulating early placental vascular changes.

\section{MATERIALS AND METHODS}

This is a descriptive comparative study. The correlation of angiogenic factor - Vascular Endothelial Growth Factor (VEGF) with recurrent abortion was evaluated in North Indian pregnant women for 60 patients with a history of Recurrent Abortion (RA) (Study group) and 60 normal pregnant women of early pregnancy (Control group) through this descriptive comparative study. The blood samples of the patients were collected to assess the serum level of VEGF by Enzyme-Linked Immunoassay (ELISA) technique Human VEGF-A BIOLISA kit. The concentration of circulating VEGF-A was evaluated by using Mann-Whitney U tests. The quantitate assay used in the present study analysed VEGF-165 isoform, which is a diffusible isoform of VEGF.

\section{RESULTS}

This variation was staggering being many times lower in RA as compared to normal early pregnant women.

\section{CONCLUSION}

The study concluded that alteration (Decreased) in the levels of VEGF may be related with RA.

\section{KEY WORDS}

Angiogenic Factor, Vascular Endothelial Growth Factor (VEGF), Recurrent Abortion (RA), Early Pregnancy, Enzyme-Linked Immunoassay.

HOW TO CITE THIS ARTICLE: Jeph RB, Agarwal S, Jeph V, et al. Serum vascular endothelial growth factor levels in normal early pregnancies and recurrent abortion patients. J. Evolution Med. Dent. Sci. 2018;7(46):4994-4996, DOI: 10.14260/jemds/2018/1111

\section{BACKGROUND}

The recurrent pregnancy loss is defined as three or more consecutive spontaneous abortions. Most of these losses are unrecognised and affect around $1 \%$ of the childbearing population. ${ }^{(1,2)}$ Adequate and appropriate vasculogenesis and angiogenesis are requirements for successful continuation of pregnancy.(3) In the initial stages of pregnancy (1 - 2 weeks), intact capillaries grow and surround the syncytiotrophoblast lacunae. These constitute the vascular supply to developing embryo.(4) VEGF is one of the most important pro-angiogenic factors, which regulate early placental vascular changes.(5)

VEGF is a soluble angiogenic factor produced by epithelial and stromal cells in upper layers of the uterine endometrium. The VEGF receptors are expressed in the endothelial cells, which can stimulate different endothelial functions. VEGF is a potent mitogen for both microvascular and macrovascular endothelial cells derived from blood vessels and lymphatics. $(6,7)$

'Financial or Other Competing Interest': None.

Submission 29-09-2018, Peer Review 23-10-2018,

Acceptance 30-10-2018, Published 12-11-2018.

Corresponding Author:

Dr. Renu Baliyan Jeph,

Vardhaman Mahavir Medical College and

SJH, New Delhi, India.

E-mail: renu.lhmc@gmail.com

DOI: $10.14260 /$ jemds/2018/1111

\section{(c) $($ ) $\odot$}

VEGF adjusts physiological and pathophysiological vascular development.(8)

VEGF is a sub-family of growth factors, which are homodimeric glycoproteins of 45,000 Daltons. VEGF-A was called just VEGF and was the first among the family to be discovered. The VEGF family comprises in of five members: VEGF-A, Placenta Growth Factor (PGF), VEGF-B, VEGF-C and VEGF-D and it exists as various isoforms. ${ }^{7,9)}$

VEGF are important signalling proteins involved in both vasculogenesis and angiogenesis, especially in the development of utero-placental circulation.

Utero-placental insufficiency is important in the pathogenesis of Intrauterine Growth Retardation (IUGR), preeclampsia and it is also considered to be a cause of various cases of recurrent abortions left unexplained.(10)

\section{MATERIALS AND METHODS}

A descriptive comparative study was conducted in the Department of Anatomy and the subjects for the study were obtained from the Department of Obstetrics and Gynaecology of Lady Hardinge Medical College and Smt. Sucheta Kriplani Hospital, New Delhi. A total of 120 pregnant subjects were included in this study, which were divided into a study group (60 subjects) and control group (60 subjects). The inclusion criteria in the study group were women in the age of $20-35$ yrs. with a history of 3 or more spontaneous abortions. Subjects had a single partner and had no anatomic, 
physiological, hormonal, chromosomal, infective or autoimmune causes for recurrent abortions. The subjects included in the control group were of the similar age group without any history of pregnancy loss and at least one live birth. Subjects with medical problems like thyroid disorders, autoimmune diseases, bleeding disorders, endocrine disease like diabetes mellitus, uterine or any other congenital abnormality were excluded.

The consent form was provided, and an informed consent was obtained from all the subjects. A detailed history, clinical examination and investigations of the subjects were recorded. Ultrasound of the pelvis was done to rule out any malformations of the genital tract, which might have been responsible for abortion. The blood was collected from the subjects to check the VEGF levels through Enzyme-Linked Immunoassay (ELISA) technique - Human VEGF-A BIOLISA kit (Diaclone). The VEGF-165 ELISA is a solid phase sandwich immunoassay designed to measure the amount of the target bound between a matched antibody pair

\section{Statistical Analysis}

The circulating VEGF-A concentration of each blood sample was obtained by calculating the mean absorbance value on the ordinate and extending a horizontal line to the standard curve. At the point of intersection, a vertical line was extended to the abscissa and the corresponding VEGF-A concentration was read. The data was statistically evaluated through the Mann-Whitney test, Median and IQR were calculated.

\section{RESULTS}

The serum VEGF levels in the study group comprising of the recurrent pregnancy loss and the control group comprising of normal pregnant females are shown below in Table 1 and Graph 1.

\begin{tabular}{|c|c|c|}
\hline Serum VEGF & Study Group & Control Group \\
\hline$(\mathrm{pg} / \mathrm{mL})$ & $\mathrm{n}=60(\%)$ & $\mathrm{n}=60(\%)$ \\
\hline $0-100$ & $30(50 \%)$ & 0 \\
\hline $100-200$ & $29(48.3 \%)$ & 0 \\
\hline $200-300$ & - & 0 \\
\hline $300-400$ & $1(1.6 \%)$ & 0 \\
\hline $400-500$ & 0 & 0 \\
\hline $500-600$ & 0 & $2(3.3 \%)$ \\
\hline $600-700$ & 0 & 0 \\
\hline $700-800$ & 0 & 0 \\
\hline $800-900$ & 0 & $3(6.6 \%)$ \\
\hline $900-1000$ & 0 & $39(65 \%)$ \\
\hline $1000-2000$ & 0 & $12(20 \%)$ \\
\hline $2000-3000$ & 0 & Table 1 \\
\hline \multicolumn{3}{|c|}{} \\
\hline
\end{tabular}

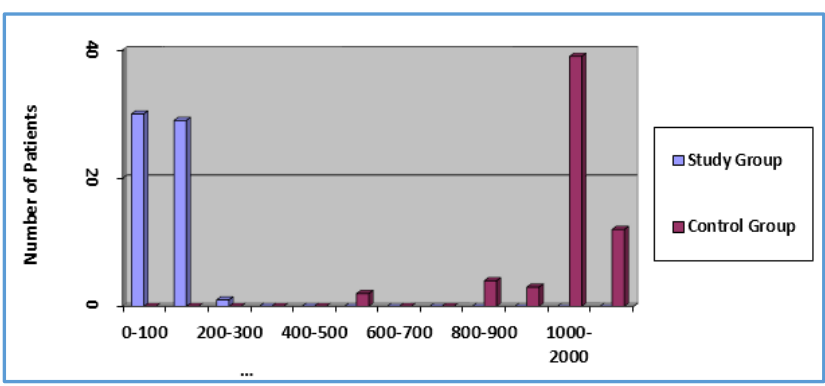

Graph 1. Distribution of patients according to Serum VEGF Levels in Study Group and Control Group

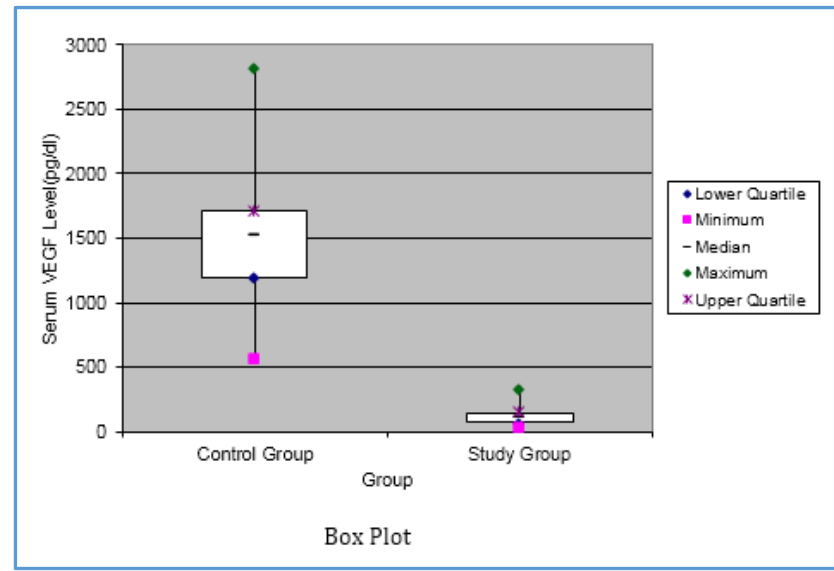

Using Mann-Whitney test, the median of Serum VEGF in control group was $1528.0 \mathrm{pg} / \mathrm{mL}$ and the median of Serum VEGF in the study group was $112.50 \mathrm{pg} / \mathrm{mL}$.

The IQR were $528 \mathrm{pg} / \mathrm{mL}$ and $79 \mathrm{pg} / \mathrm{mL}$ for the control group and study group respectively.

\section{DISCUSSION}

In the present prospective study, it was found that high levels of serum VEGF were seen in the serum of the normal pregnant females in comparison to the serum VEGF levels of females with Recurrent Pregnancy Loss. In many other studies, the VEGF levels were found to be higher in the Recurrent Pregnancy Loss females in comparison to the normal pregnant females. In the present study, free VEGF was determined while in most of the other studies total VEGF was studied. The lower levels in the present study are probably due to this. The decrease in the levels of VEGF patients can be explained on the basis of hypothesis involving pro-angiogenic and anti-angiogenic factors.

Recurrent abortions characterised by inflammatory infiltrates in the placenta cause defective placental development, which is the main site of VEGF production and thus can lead to decreased production of VEGF which increases the chances of abortion. One more mechanism can be that the Anti-angiogenic soluble VEGFR1 (SFlt-1) gets elevated and inhibits the action of VEGF by binding free VEGF and rendering it inactive. Thus maybe both VEGF and SFlt-1 plays an important role in the pathogenesis of RSA.

Angiogenesis is very important for the endometrium development, embryo implantation and pregnancy. Increased levels of VEGF is observed around the pre-implantation period. It cooperates with multiple endothelial growth factors to regulate the formation of new blood vessels and vascular function in the endometrium. ${ }^{1}$

VEGF also maintain newly formed capillaries, induces vascular permeability and macrophage chemotaxis. Any alteration in the levels of this factor can cause abnormal angiogenesis in endometrium and might lead to failure in implantation and recurrent miscarriage. $(11,4,6)$

This current case-control study conducted on North Indian population to evaluate the levels of serum VEGF in normal early pregnancies and recurrent abortions. In the present study, it was found serum VEGF concentration increases with the period of gestation during the first trimester. It has also been found in other studies. Moleskness et al, 2004 reported a significant rise in the level of VEGF during early pregnancy.(12) 
In the present study, the mean VEGF concentration in the patients with recurrent abortion was staggering 14 times lower in comparison to normal pregnant women. Wheeler's also reported an increasing value of serum VEGF with the period of gestation.(13) Evan et al reported a consistent rise in maternal serum VEGF levels during the first trimester of pregnancy.(14) Few studies have also reported the serum VEGF levels to be significantly higher in recurrent spontaneous abortions in comparison to normal pregnancy.(15) It was also found that the mean serum VEGF increased in the second trimester in comparison to the first trimester.

There are few studies that have focused on the expression of VEGF. Banerjee et al, 2013 observed lower VEGF proteins expression in the endometrium in women with recurrent abortions.(16) Lash et al also found reduced expression of VEGF and VEGFR-2 in cases of recurrent abortions.(17) There is another study done by Vuorela et al, 2000 observed decreased expression of VEGF and VEGF receptors in decidua and villous tissue samples from recurrent pregnancy abortion cases.(18)

VEGF contributes to the mature functioning blood vessels, decreased apoptosis of endothelial cells. Decreased production of VEGF leads to deregulation of the balance between endothelial and epithelial cells' apoptosis and proliferation. This results in a defect in embryogenesis and damage to mature tissue. $(19,20)$

Therefore, it has been observed that the reduced capability of normal tissue to regulate VEGF under stressful conditions like placenta formation will lead to the formation of a blood vessel network, which has a very high apoptotic tendency. This explains detachment of placenta and spontaneous abortions. Reduced expression of VEGF has been linked with spontaneous miscarriages likely due to defective factor and placental angiogenesis.(21) This indicates that there is a relationship between early recurrent spontaneous abortion and VEGF and low levels of VEGF may be associated with the pathogenesis of recurrent pregnancy abortion.

\section{CONCLUSION}

The present study suggests that alteration in the levels of VEGF contribute to recurrent miscarriages. Although, the definitive role of VEGF in the pathogenesis of recurrent pregnancy loss needs to be further investigated.

\section{REFERENCES}

[1] Stirrat GM. Recurrent miscarriages. Lancet 1990;336(8716):673-5.

[2] Li TC, Makris M, Tomus $M$, et al. Recurrent miscarriages: aetiology, management and prognosis. Human Reproduction Update 2002;8(5):463-81.

[3] Te Velde EA, Exalto N, Hesseling P, et al. First trimester development of human chorionic villous vascularization studies with CD34 immunohistochemistry. Hum Reprod 1997;12(7):1577-81.

[4] Nardo LG. Vascular endothelial growth factor expression in the endometrium during the menstrual cycle, implantation window and early pregnancy. Current Opinion in Obstet \& Gynaecol 2005;17(4):419-23
[5] Gargett CE, Rogers PA. Human endometrial angiogenesis. Reproduction 2001;121(2):181-6.

[6] Ferrara N, Davis-Smyth T. The biology of vascular endothelial growth factor. Endocrine Reviews 1997;18(1):4-25.

[7] Neufeld G, Cohen T, Gengrinovitch S, et al. Vascular endothelial growth factor (VEGF) and its receptors. FASEB J 1999;13(1):9-22.

[8] Pang L, Wei Z, Li O, et al. An increase in vascular endothelial growth factors (VEGF) and VEGF soluble receptors-1(sFlt-1) are associated with early recurrent spontaneous abortion. PLoS One 2013;8(9):e75759.

[9] Clauss M. Molecular biology of the VEGF and the VEGF receptor family. Semin Thromb Hemost 2000;26(5):561-9.

[10] Norwitz ER. Defective implantation and placentation: laying the blueprint for pregnancy complications. Reprod Biomed Online 2006;13(4):591-9.

[11] Sharkey A. Cytokines and implantation. Rev Reprod 1998;3(1):52-61.

[12] Molskness TA, Stouffer RL, Burry KA, et al. Circulating levels of free and total VEGF-A, soluble VEGFR-1 and VEGFR-2 and angiogenin during ovarian stimulation in non-human primates and women. Human Reproduction 2004;19(4):822-30.

[13] Wheeler T, Evans PW, Anthony FW, et al. Relationship between maternal serum vascular endothelial growth factor concentration in early pregnancy and fetal \& placental growth. Human Reproduction 1999;14(6):1619-23.

[14] Evan P, Wheeler T, Anthony F, et al. Maternal serum vascular endothelial growth factor during early pregnancy. Clinical Science (Lond) 1997;92(6):567-71.

[15] Amirchanghmaghi E, Rezaci A, Moini A, et al. The role of VEGF receptors in recurrent receptors in recurrent spontaneous abortion. Iranian Journal of Reproduction Medicine 2013: p. 15.

[16] Banerjee P, Jana SK, Pasricha P, et al. Proinflammatory cytokines induced altered expression of cyclooxygenase- 2 gene results in unreceptive endometrium in women with idiopatheic recurrent spontaneous miscarriages. Fertil Steril 2013;99(1):179-87.

[17] Lash GE, Innes BA, Drury JA, et al. Localization of angiogenic growth factor and their receptors in the human endometrium throughout the menstrual cycle and in recurrent miscarriages. Hum Reprod 2012;27(1):183-95.

[18] Vuorela P, Carpen 0, Tulppala M, et al. VEGF, its receptors and the tie receptors in recurrent misscarriage. Mol Hum Reprod 2000;6(3):276-82.

[19] Print C, Valtola R, Evans A, et al. Soluble factors from human endometrium promote angiogenesis and regulate the endothelial cell transcriptome. Hum Reprod 2004;19(10):2356-66

[20] Zygmunt M, Herr F, Munstedt K, et al. Angiogenesis and vasculogenesis in pregnancy. Eur J Obstet Gynecol Reproductive Biology 2003;110(Suppl 1):S10-S8.

[21] Almawi WY, Saldanha FL, Mahmood NA, et al. Relationship between VEGFA polymorphisms and serum VEGF protein levels and recurrent spontaneous miscarriages. Human Reprod 2013;28(10):2628-35. 